\title{
Hop, Skip and Jump - What Are Modern "Jump" Tests Finding in Stock Returns?
}

\author{
Michael William Schwert
}

Professor George Tauchen, Faculty Advisor

Honors Thesis submitted in partial fulfillment of the requirements for Graduation with Distinction in Economics in Trinity College of Duke University.

Duke University

Durham, North Carolina

2009 


\section{Acknowledgements}

I would like to thank George Tauchen for leading the Honors Finance Seminar and for his advice throughout the development of this paper. I would also like to thank the members of the Honors Finance Seminar for their comments and feedback on my work. Additionally, I am grateful to Tim Bollerslev, Bjorn Eraker, Andrey Fradkin, George Jiang, Roel Oomen, and G. William Schwert for their helpful suggestions. 


\begin{abstract}
This paper applies several jump detection tests to intraday stock price data sampled at various frequencies. It finds that the choice of sampling frequency has an effect on both the amount of jumps detected by these tests, as well as the timing of those jumps. Furthermore, although these tests are designed to identify the same phenomenon, they find different amounts and timing of jumps when performed on the same data. These results suggest that these jump detection tests are probably identifying different types of jump behavior in stock price data, so they are not really substitutes for one another.
\end{abstract}




\section{Introduction}

In recent years there has been a great deal of interest in studying jumps in asset price movements. Reasons why it is important to know when and how frequently jumps occur include risk management and the pricing and hedging of derivative contracts. Investors would benefit greatly from knowing the properties of jumps, since large instantaneous drops in asset prices result in large instantaneous losses. The effect of jumps on derivative pricing is equally significant, especially considering the important role derivatives play in modern financial markets. When asset price movements are continuous, investors can perfectly hedge derivative contracts, but when jumps occur, they cause a change in the derivative price that is non-linear to the change in the price of the underlying asset. Thus, jumps introduce an unhedgeable risk to the holders of derivative contracts.

The ability to identify realized jumps in the financial markets could provide helpful information such as how frequently jumps occur, how large the jumps are, and whether they tend to occur in clusters. With this goal in mind, several authors have developed tests to determine whether or not an asset price movement is a statistically significant jump. These tests take advantage of the high-frequency intraday price data available today through electronic sources. Barndorff-Nielsen and Shephard $(2004,2006)$ use the difference between an estimate of variance and a jump-robust measure of variance to detect jumps over the course of a day. Approaching the problem differently, Jiang and Oomen (2007) exploit high order sample moments of returns to identify days that include jumps. Aït-Sahalia and Jacod (2008) also exploit high order sample moments of returns to detect jumps by comparing price data sampled at two different frequencies. Lee and Mykland (2008) test for jumps at individual price observations by scaling returns by a local volatility measure. While these tests employ different strategies for detecting jumps, they are all designed to identify the same phenomenon.

Due to the distortion of jump test statistics by market microstructure noise ${ }^{1}$ in the highest-frequency intraday price data, it is necessary to sample prices at a lower frequency. There is a range of sampling frequencies in which the effect of market microstructure noise is minimal and the informational content of high-frequency data is maintained. When performed on price data sampled at different frequencies within this range, one might expect

\footnotetext{
${ }^{1}$ Market microstructure noise is explained in Section 2.2.
} 
that these tests would find similar results, given that all samples are based on the same original data. However, this paper finds that this is not necessarily the case, as the choice of sampling frequency affects both the number of jumps detected by each test and the timing of those jumps. These findings raise questions about the types of jumps that are being identified by these different tests.

The remainder of this paper is organized as follows. Section 2 introduces the model of equity returns that forms the basis of the jump tests. Included in this section is a discussion of market microstructure noise. Section 3 describes several of the jump tests developed over the past few years. Section 4 discusses the high-frequency stock price data used in this paper. Section 5 discusses the results of a relative comparison of the jump tests described in Section 3. Section 6 interprets these results and puts them in context with the jump literature. Finally, Section 7 summarizes the paper and highlights important conclusions.

\section{Model of Equity Returns}

Before discussing the jump tests and results, it is important to understand the theoretical model of asset price movements and the concept of market microstructure noise. Section 2.1 introduces the stochastic price process on which the jump tests in Section 3 are based. Section 2.2 explains the market microstructure noise that creates the necessity to sample prices at frequencies lower than the highest available.

\subsection{Stochastic Model of Returns and Volatility}

This paper uses a model of asset price movements that includes jumps in the price evolution. The following stochastic differential equation, discussed by Merton (1971), is a continuous model of logarithmic price movements:

$$
d p(t)=\mu(t) d t+\sigma(t) d W(t), 0 \leq t \leq t_{n}=T,
$$

where $\mu(t) d t$ represents the time-varying drift component and $\sigma(t) d W(t)$ represents the timevarying volatility component of the asset price, and $W(t)$ is a standard Brownian motion. The inclusion of Brownian motion in this model contributes randomness to price movements. This is appropriate, given Fama's (1965) finding that stock prices follow a random walk. 
Given recent findings in the academic finance literature that suggest there are discontinuities in asset price movements, it is appropriate to add a jump component to the model of logarithmic prices:

$$
d p(t)=\mu(t) d t+\sigma(t) d W(t)+\kappa(t) d q(t), \quad 0 \leq t \leq t_{n}=T .
$$

Merton (1976) introduced this equation where the discontinuous component of the price process is $\kappa(t) d q(t)$. Here, $q(t)$ is a counting process and $\kappa(t)$ is the magnitude of the jump. It is commonly assumed that $q(t)$ is a Poisson process, so the model produces rare large jumps.

While it is fairly simple to estimate the drift and volatility components of the price process, it is more difficult to estimate the jump component. Several authors have developed tests to determine when a price movement is probably a jump, as opposed to a large continuous movement. It is worth noting that one can never be certain that a price movement is a jump, as one can only observe prices at discrete intervals and cannot form a continuous picture of the price path.

\subsection{Market Microstructure Noise}

A common method for calculating the theoretical price of an equity is to sum the discounted value of all future dividend payments. The constant dividend growth model assumes that the firm's dividend payout ratio is constant over time and that the firm's expected earnings per share grow by a constant yearly growth rate $g$. The expected value of the dividend payout in year $i$ is

$$
E\left(D_{i}\right)=D_{0}(1+g)^{i}=E_{0} d(1+g)^{i},
$$

where $E_{0}$ is the firm's current level of annual earnings per share and $d$ is the firm's dividend payout ratio. Based on this expectation, the firm's current stock price is

$$
P_{0}=\sum_{i=0}^{\infty} \frac{E\left(D_{i}\right)}{r^{i}}=\frac{D_{0}(1+g)}{r-g},
$$

where $r$ is the required rate of return as determined by the Capital Asset Pricing Model. For the remainder of this paper, the price of a stock as defined in (2.2.2) is referred to as the fundamental price.

The model of market microstructure noise describes the deviation of the observed stock prices from their fundamental values. The observed price of an equity at time $t$ is 


$$
p^{*}(t)=p(t)+\varepsilon_{t},
$$

where $p(t)$ is the logarithm of the fundamental price. The evolution of $p(t)$ is described by (2.2.2). In this equation, $\varepsilon_{t}$ represents a short-term deviation from the fundamental price called market microstructure noise. Note that this noise term is added after taking the logarithm of the price, so it is proportional to the stock price.

Microstructure noise results from market frictions, such as bid-ask bounce, which cause the price to deviate briefly from its fundamental value. Barndorff-Nielsen and Shephard (2005) suggest using mid-quote ${ }^{2}$ data to mitigate the effect of bid-ask bounce, but this is impossible when full-quote data are unavailable. This paper uses transaction data, where trades occur at either the bid price or the ask price ${ }^{3}$, so bid-ask bounce creates noise in the high-frequency price series. Market microstructure noise is noticeable when estimating variance and other statistics and can have a significant impact on tests for jumps in asset prices. Section 5 discusses a potential solution to this problem. Note that this paper does not consider possible medium-term deviations of stock prices from their fundamental values, such as those discussed by Fama and French (1988).

\section{Jump Tests}

In recent years a number of papers have been published introducing new statistical tests to detect discontinuities in the price process. Before examining and comparing these tests, it is necessary to provide some background on the tests and how they detect jumps in asset price movements. Section 3.1 explains the first jump tests introduced to the literature, the Barndorff-Nielsen Shephard $(2004,2006)$ tests. Section 3.2 discusses tests created by Jiang and Oomen (2007), who were the first authors to attempt to correct the effects of market microstructure noise. Section 3.3 describes Lee and Mykland's (2008) test that checks for a jump at every price observation, rather than checking if a jump occurs within a particular sample period, such as a day. Finally, Section 3.4 explains Aït-Sahalia and Jacod's (2008) test, which compares calculations of higher moments at two different sampling frequencies to detect jumps over the course of a day.

\footnotetext{
${ }^{2}$ With full-quote data, one can average the bid and the ask at each quote to create a mid-quote price series.

${ }^{3}$ Many trades occur between the bid and the ask because of order matching. However, these transactions may still deviate from the fundamental price, contributing to market microstructure noise.
} 


\subsection{Barndorff-Nielsen Shephard Tests}

Barndorff-Nielsen and Shephard $(2004,2006)$ developed a test that uses high-frequency price data to determine whether there is a jump over the course of a day. Their test compares two measures of variance: Realized Variance, which converges to the integrated variance plus a jump component as the time between observations approaches zero; and Bipower Variation, which converges to the integrated variance as the time between observations approaches zero, and is robust to jumps in the price path, an important fact for this application. The integrated variance of a price process is the integral of the square of the $\sigma(t)$ term in (2.2.2), taken over the course of a day. Since prices cannot be observed continuously, one cannot calculate integrated variance exactly, and must estimate it instead.

For the rest of this paper, $P\left(t_{i}\right)$ is the stock price at time $t_{i}$, and

$$
r_{i}=\log \left(\frac{P\left(t_{i}\right)}{P\left(t_{i-1}\right)}\right)
$$

is the geometric return from time $t_{i-1}$ to time $t_{i}$. Then, Realized Variance and Bipower Variation are described by the following equations:

$$
\begin{gathered}
R V=\sum_{i=2}^{n}\left|r_{i}\right|^{2} \underset{n \rightarrow \infty}{\longrightarrow} \int_{0}^{T} \sigma^{2}(v) d v+\sum_{i=1}^{n} \kappa^{2}\left(t_{i}\right) q\left(t_{i}\right), \\
B V=\frac{\pi}{2}\left(\frac{n}{n-1}\right) \sum_{i=3}^{n}\left|r_{i}\right|\left|r_{i-1}\right| \underset{n \rightarrow \infty}{\longrightarrow} \int_{0}^{T} \sigma^{2}(v) d v,
\end{gathered}
$$

where $n$ is the number of price observations in a day. The limits in (3.1.2) and (3.1.3) describe the infill asymptotics of the Realized Variance and Bipower Variation, meaning that as the number of observations within a day approaches infinity, the interval at which observations are made approaches zero and the period over which observations are made is held constant.

Huang and Tauchen (2005) also consider Relative Jump, a measure that approximates the percentage of total variance attributable to jumps:

$$
R J=\frac{R V-B V}{R V} .
$$

This statistic approximates the ratio of the sum of squared jumps to the total variance and is useful because it scales out long-term trends in volatility so one can compare the relative contribution of jumps to the variance of two price series with different volatilities. 
To develop a statistical test to determine whether there is a significant difference between $R V$ and $B V$, one needs an estimate of integrated quarticity. Andersen, Bollerslev, and Diebold (2004) recommend using a jump-robust realized Tri-Power Quarticity,

$$
T P=n \mu_{4 / 3}^{-3}\left(\frac{n}{n-2}\right) \sum_{i=4}^{n}\left|r_{i}\right|^{4 / 3}\left|r_{i-1}\right|^{4 / 3}\left|r_{i-2}\right|^{4 / 3} \underset{n \rightarrow \infty}{\longrightarrow} \int_{0}^{T} \sigma^{4}(v) d v,
$$

where

$$
\mu_{m}=E\left|u^{m}\right|, \quad u \sim N(0,1) .
$$

Barndorff-Nielsen and Shephard recommend the realized Quad-Power Quarticity,

$$
Q P=n \mu_{1}^{-4}\left(\frac{n}{n-3}\right) \sum_{i=5}^{n}\left|r_{i}\right|\left|r_{i-1}\right|\left|r_{i-2}\right|\left|r_{i-3}\right| \underset{n \rightarrow \infty}{\longrightarrow} \int_{0}^{T} \sigma^{4}(v) d v .
$$

There are numerous potential test statistics that use these estimates of integrated variance and integrated quarticity, but Huang and Tauchen find that these two asymptotically standard normal test statistics perform best in simulations:

$$
\begin{aligned}
& z_{T P-\max }=\frac{R J}{\sqrt{\left(\left(\frac{\pi}{2}\right)^{2}+\pi-5\right)\left(\frac{1}{n}\right) \max \left(1, \frac{T P}{B V^{2}}\right)}}, \\
& z_{Q P-\max }=\frac{R J}{\sqrt{\left(\left(\frac{\pi}{2}\right)^{2}+\pi-5\right)\left(\frac{1}{n}\right) \max \left(1, \frac{Q P}{B V^{2}}\right)}} .
\end{aligned}
$$

The null hypothesis that there are no jumps in a day is rejected at a .01 level of significance if the $z$-statistic is greater than 2.33 .

\subsection{Jiang Oomen "Swap Variance" Tests}

Following up on the work of Barndorff-Nielsen and Shephard, Jiang and Oomen (2007) proposed their own tests for the presence of jumps in asset prices. Their "Swap Variance" tests $^{4}$ can intuitively be thought of as measuring the impact of jumps on high order moments of returns by taking the difference between the accumulated arithmetic and geometric returns over the course of a day. Arithmetic and geometric returns are defined as follows:

\footnotetext{
${ }^{4}$ They call their tests the "Swap Variance" tests because $S w V$ is related to the profit/loss function of a variance swap replication strategy using a "log contract" (p. 2).
} 


$$
\begin{array}{ll}
\text { Arithmetic return: } & R_{i}=\frac{P\left(t_{i}\right)-P\left(t_{i-1}\right)}{P\left(t_{i-1}\right)}, \\
\text { Geometric return: } & r_{i}=\log \left(\frac{P\left(t_{i}\right)}{P\left(t_{i-1}\right)}\right) .
\end{array}
$$

The tests rely on a statistic they call "Swap Variance," which is equal to the difference between accumulated arithmetic and geometric returns:

$$
S w V=2 \sum_{i=2}^{n}\left(R_{i}-r_{i}\right) \text {. }
$$

The tests use the difference between Swap Variance and Realized Variance to detect jumps. A Taylor series expansion of this difference provides further understanding of how these tests exploit higher order returns to check for jumps:

$$
S w V-R V=\frac{1}{3} \sum_{i=2}^{n}\left[r_{i}\right]^{3}+\frac{1}{12} \sum_{i=2}^{n}\left[r_{i}\right]^{4}+\ldots
$$

There will be a drastic difference between the value of this sum when jumps are present and the value when there are no jumps over the course of a day, due to the influence of large exponents of the return. Based on this idea, the authors created statistical tests of whether this value is significant. The $z$-statistics that test the null hypothesis of no jumps in a day are as follows:

$$
\begin{gathered}
\text { Difference test: } \quad J O_{D i f f}=\frac{n}{\sqrt{\Omega_{S w V}}}(S w V-R V), \\
\text { Logarithmic test: } \quad J O_{L o g}=\frac{n V}{\sqrt{\Omega_{S w V}}}(\log (S w V)-\log (R V)), \\
\text { Ratio test: } \quad J O_{\text {Ratio }}=\frac{n V}{\sqrt{\Omega_{S w V}}}\left(1-\frac{R V}{S w V}\right),
\end{gathered}
$$

where

$$
\Omega_{S w V}=\frac{\mu_{6}}{9} \frac{n^{3} \mu_{3 / 2}^{-4}}{n-3} \sum_{i=5}^{n}\left|r_{i}\right|^{3 / 2}\left|r_{i-1}\right|^{3 / 2}\left|r_{i-2}\right|^{3 / 2}\left|r_{i-3}\right|^{3 / 2}
$$

and $V$ is the integrated variance, which one can estimate well with the Bipower Variation calculated with prices sampled at a frequency insensitive to market microstructure noise. Section 5 introduces techniques that indicate what frequency would be appropriate to use for 
this calculation. These scaling factors are necessary for the $J O$ statistics to be distributed standard normal.

As a modification to their "Swap Variance" tests, Jiang and Oomen introduce a novel approach to the jump detection literature by creating tests that account for the effect of i.i.d. market microstructure noise. The Microstructure Noise Robust $z$-statistics are quite similar to the ones above, but the $\Omega_{S w V}$ term is estimated with a market microstructure noise correction.

$$
\begin{gathered}
\text { Difference test: } M N R_{D i f f}=\frac{S w V-R V}{\sqrt{\Omega_{S w V}^{*}}}, \\
\text { Logarithmic test: } \quad M N R_{\text {Log }}=\frac{V^{*}}{\sqrt{\Omega_{S w V}^{*}}}(\log (S w V)-\log (R V)), \\
\text { Ratio test: } \quad M N R_{\text {Ratio }}=\frac{V^{*}}{\sqrt{\Omega_{S w V}^{*}}}\left(1-\frac{R V}{S w V}\right), \\
V^{*}=V+2 n \omega^{2},
\end{gathered}
$$

where

with $V$ estimated by the Bipower Variation of prices sampled at a 10-minute interval and

$$
\Omega_{S w V}^{*}=4 n \omega^{6}+12 \omega^{4} V+\frac{8}{n} \omega^{2} Q+\frac{5}{3 n^{2}} X,
$$

where $\omega^{2}$ is an estimate of the variance of the market microstructure noise, $V$ is an estimate of integrated variance, $Q$ is an estimate of integrated quarticity, and $X$ is an estimate of integrated sexticity. Thus, to calculate the test statistics, estimates of these variables are necessary.

Roll (1984) introduced the idea of using the first order negative serial covariance of returns as an estimate of the amount of bid-ask spread over the course of a day. Bid-ask spread is a major contributor to market microstructure noise, so Jiang and Oomen deem it appropriate to estimate the variance of noise with the first order negative serial correlation of returns,

$$
\omega^{2}=-\frac{1}{n-1} \sum_{i=3}^{n} \log \left(\frac{S\left(t_{i}\right)}{S\left(t_{i-1}\right)}\right) \log \left(\frac{S\left(t_{i-1}\right)}{S\left(t_{i-2}\right)}\right) .
$$

They introduce the Noise-corrected Bipower Variation as an estimate of integrated variance:

$$
V \approx B V^{*}=\frac{B V}{1+c_{b}(\gamma)}
$$


where

$$
\begin{gathered}
c_{b}(\gamma)=(1+\gamma) \sqrt{\frac{1+\gamma}{1+3 \gamma}}+\gamma \frac{\pi}{2}-1+2 \frac{\gamma}{(1+\lambda) \sqrt{2 \lambda+1}}+2 \gamma \pi \kappa(\lambda), \\
\gamma=\frac{n \omega^{2}}{\bar{V}}, \\
\lambda=\frac{\gamma}{1+\gamma}, \\
\kappa(\lambda)=\int_{-\infty}^{\infty} x^{2} \Phi(x \sqrt{\lambda})(\Phi(x \sqrt{\lambda})-1) \phi(x) d x
\end{gathered}
$$

and $\bar{V}$ is estimated by the Bipower Variation of prices sampled at a 10-minute interval. Jiang and Oomen note that estimating the integrated quarticity and sexticity is troublesome using noisy data, so they suggest using the squared and cubed estimates of the integrated variance to estimate $Q$ and $X$, respectively.

\subsection{Lee Mykland Test}

While most of the jump tests developed over the past several years test for jumps during a set span of time, Lee and Mykland (2008) developed a test that checks for a jump at each individual observation. Their test calculates the ratio of the return at each price observation to a measure of "instantaneous volatility" over a period preceding that observation. If this ratio exceeds a certain threshold, they identify the observation as a jump. The statistic $L(i)$ that tests for a jump at time $t_{i}$ is defined as

$$
L(i)=\frac{r_{i}}{\hat{\sigma}\left(t_{i}\right)}
$$

where

$$
\hat{\sigma}\left(t_{i}\right)^{2}=\frac{1}{K-2} \sum_{j=i-K+2}^{i-1}\left|r_{j}\right|\left|r_{j-1}\right| .
$$

and $r_{i}$ is the return as defined in Section 3.1.

The instantaneous volatility measure is similar to Bipower Variation as defined in Section 3.1, with a different scaling constant, so this test is robust to the presence of jumps in prior periods. Lee and Mykland suggest that it is appropriate to choose the window size $K$ between $252 \times n$ and $\sqrt{252 \times n}$, where $n$ is the number of observations in a day. For this paper, I use $350,250,150$, and 100 as values of $K$ for the 1 -minute, 5 -minute, 10 -minute, and 
15-minute sampling intervals, respectively. These window sizes are long enough that instantaneous volatility maintains the quality of being a jump-robust Bipower Variation, and short enough that it effectively scales the test statistic for trends in volatility.

The asymptotic distribution of the test statistic is as follows:

$$
\frac{|L(i)|-C_{n}}{S_{n}} \rightarrow \xi,
$$

where

$$
P(\xi \leq x)=\exp \left(-e^{-x}\right) .
$$

The constants

$$
\begin{gathered}
C_{n}=\frac{(2 \log n)^{1 / 2}}{c}-\frac{\log \pi+\log (\log n)}{2 c(2 \log n)^{1 / 2}}, \\
S_{n}=\frac{1}{c(2 \log n)^{1 / 2}}, \\
c=\sqrt{\frac{2}{\pi}}
\end{gathered}
$$

scale the $L(i)$ statistic to be exponentially distributed.

The threshold for rejecting the null hypothesis of no jump for an individual observation at a .01 significance level is 4.6001 . For the purpose of comparing this test to other tests, I want to use this test to detect jumps over the course of a day. However, using a .01 level of significance to reject the null hypothesis of no jump at each observation is not equivalent to using a .01 level of significance to reject the null hypothesis of no jumps in a day. Thus, to use this statistic for determining whether there is a jump over the course of a day, I derive rejection thresholds for performing the Lee Mykland test on price data sampled at a variety of intervals. Here I assume that each observation is independent and identically distributed, so the test at each observation is independent of tests at other observations. Thus, the threshold for rejecting the null hypothesis of no jumps over the course of a day is $x$, where

$$
P(\xi \leq x)^{n}=\exp \left(-e^{-x}\right)
$$

and $n$ is the number of observations in a day. These threshold values are 10.554, 8.944, 8.264 , and 7.858 for the 1-minute, 5-minute, 10-minute, and 15-minute sampling intervals, respectively. 


\subsection{Aït-Sahalia Jacod Test}

Aït-Sahalia and Jacod (2008) recently introduced a test that takes advantage of higher return moments to detect jumps in asset price processes. Their test takes the sum of absolute returns to a power $p$ ( $p=4$ here) over the course of a day at two different sampling intervals, $k \Delta$ and $\Delta$, and uses the ratio of these sums to check if there are jumps in the day. $\Delta$ is called the base sampling interval for the remainder of this paper. The test statistics are

$$
\begin{gathered}
\hat{B}(p, \Delta)=\sum_{i=1}^{n}\left|\log \left(\frac{S\left(t_{i \Delta}\right)}{S\left(t_{(i-1) \Delta}\right)}\right)\right|^{p} \\
A S J(p, k, \Delta)=\frac{\hat{B}(p, k \Delta)}{\hat{B}(p, \Delta)},
\end{gathered}
$$

where $n$ is the total number of observations available in a day (385 in the minute-by-minute stock price data). Due to the use of higher order returns, when $p>2, \hat{B}(p, \Delta)$ eliminates the continuous component of returns as the number of observations approaches infinity and emphasizes the jump component. Thus, the $A S J$ statistic approaches 1 when jumps are present, because regardless of sampling frequency, $\hat{B}(p, \Delta)$ will approach the same number. When there are no jumps in the day, $\hat{B}(p, \Delta)$ and $\hat{B}(p, k \Delta)$ will both approach zero, but at different rates, so the $A S J$ statistic will converge to $k^{p / 2^{-1}}$. These limits are the basis of this jump test.

The null hypothesis of no jumps is rejected when $A S J<\xi$, where

$$
\begin{gathered}
\xi=k^{p / 2-1}-z_{\alpha} \sqrt{\hat{V}} \\
\text { with } \hat{V}=\frac{\Delta M(p, k) \hat{A}(2 p, \Delta)}{\hat{A}(p, \Delta)^{2}} \\
\text { and } \left.\quad \hat{A}(p, \Delta)=\frac{\Delta^{1-p / 2}}{\mu_{p}} \sum_{i=1}^{n}\left|\log \left(\frac{S\left(t_{i \Delta}\right)}{S\left(t_{(i-1) \Delta}\right)}\right)\right|^{p} 1_{\left\{\left|\log \left(\frac{S\left(t_{i \Delta}\right)}{S\left(t_{(i-1) \Delta}\right)}\right)\right|<\alpha \Delta^{\sigma}\right.}\right\} .
\end{gathered}
$$

For the choice of $p=4, M(4, k)=\frac{16 k\left(2 k^{2}-k-1\right)}{3}$, and the authors suggest using $\alpha=0.05$ and $\varpi=0.48$ as the other constants, as the test performs well in simulations under these conditions. The results in Table 2 come from performing the test using $\alpha=0.05$ 
$\left(z_{\alpha}=1.64\right)$, but for the purpose of directly comparing jump days with other authors' tests, the test is performed using $\alpha=0.01\left(z_{\alpha}=2.33\right)$ as the level of significance for the results in Tables 3 and 4 .

\section{Data}

The great wealth of high-frequency financial data available to researchers today through online resources is responsible for the recent interest in high-frequency jump detection techniques. This paper uses price data for the 10 stocks in the S\&P 100 Index with the largest market capitalization at the end of 2007, excluding Apple and Google, for which there are a limited number of days available. This sample includes ExxonMobil (NYSE: XOM), General Electric (NYSE: GE), Microsoft (NASDAQ: MSFT), AT\&T (NYSE: T), Procter \& Gamble (NYSE: PG), Chevron (NYSE: CVX), Johnson \& Johnson (NYSE: JNJ), Bank of America (NYSE: BAC), Cisco Systems (NASDAQ: CSCO), and Altria Group (NYSE: MO).

These price data were downloaded from a commercial data vendor, price-data.com, and are collected every minute from 9:35 AM until 4:00 PM every trading day from 1997 to 2007. The number of trading days available for each of these stocks ranges from 1566 to 2686 days. This difference in length of sample period is due to certain stocks that did not trade until after 1997, such as ExxonMobil and Chevron, which first traded following a merger in 1999 and a ticker change in 2001, respectively. Additional Table 1 in the Appendix contains information on the sample of equities used for the calculations in this report.

\section{Relative Comparison of Jump Tests}

This section contains the results of performing the jump tests with prices sampled at different frequencies and comparisons of these results. First, Section 5.1 motivates the idea of sampling prices at different frequencies for the jump tests. Then, Section 5.2 discusses the results of the jump tests performed at different sampling frequencies, and the surprising incoherence among those results. Finally, Section 5.3 discusses the results of a direct comparison of the jump tests and the incoherence that also exists among these tests. 


\subsection{Determining the Proper Sampling Frequency}

As discussed in Section 2.2, the effect of market microstructure noise must be taken into account when calculating statistics for jump tests. Market microstructure noise appears to have a significant impact on the Barndorff-Nielsen Shephard $Z_{T P \text {-max }}$ and $Z_{Q P \text {-max }}$ tests performed on minute-by-minute price data. Across the ten stocks used in this paper, these tests detect an average of $49.29 \%$ and $53.29 \%$ of all days as having jumps at a .01 level of significance. Given the underlying model of large, infrequent jumps, these percentages seem rather high. It is likely that market microstructure noise is influencing the test at this high of a sampling frequency.

Confronted with the problem of determining the effect of market microstructure noise and finding the optimal sampling frequency at which to calculate Realized Variance, Anderson, Bollerslev, Diebold and Labys (1999) developed a graphical tool called the volatility signature plot. The volatility signature plot graphs the effect of sampling frequency on volatility, with sampling interval ${ }^{5}$ on the horizontal axis and volatility on the vertical axis.

The key to the volatility signature plot is that the variance of a price process is independent of the frequency at which prices are observed, so long as prices adhere to the semi-martingale process described in (2.1.1). However, as market microstructure noise causes the price to deviate from its fundamental value as described in (2.2.2), it distorts the calculation of variance estimates like Realized Variance and Bipower Variation. To better understand the Barndorff-Nielsen Shephard results, I construct volatility signature plots for Realized Variance and Bipower Variation.

Anderson et al. find that the highest levels of volatility occur at the highest sampling frequencies for a liquid asset, and that the lowest levels of volatility occur at the highest sampling frequencies for an illiquid asset. Thus, the volatility signature plot for the liquid asset is a downward sloping curve and the volatility signature plot for the illiquid asset is an upward sloping curve. As the assets in this paper are stocks that trade extremely frequently, their volatility signature plots should be similar to that of the highly liquid asset.

The plots for Realized Variance (Figure 1) and Bipower Variation (Figure 2) are fairly difficult to interpret in the context of the Barndorff-Nielsen Shephard tests, but as

\footnotetext{
5 e.g.: prices sampled at a 5-minute interval have observations at 9:35, 9:40, 9:45, .., a total of 77 daily observations
} 
expected, the value of each variance measure decreases as the sampling interval increases, particularly in the 1-minute to 5-minute segment of the graph. For the Microstructure Noise Robust Jiang Oomen tests, it seems appropriate to sample prices at a 10-minute interval when estimating the integrated variance, given the relatively high levels of Bipower Variation at the highest sampling frequencies.

To further examine the effect of market microstructure noise on the BarndorffNielsen Shephard results above, I plot the relation between Relative Jump and sampling frequency for the ten stocks, employing the idea behind the volatility signature plot. Interestingly, this Relative Jump signature plot (Figure 3) is more revealing than the volatility signature plots in Figures 1 and 2. In this plot, Relative Jump decreases drastically as the sampling frequency decreases from the highest levels, stabilizes between the 5-minute and 11-minute sampling intervals, and begins increasing at lower frequencies, implying the emergence of sampling error. Given this information, it seems appropriate to sample prices at frequencies in the flat portion of the plot when performing the Barndorff-Nielsen Shephard tests on this data. I also sample prices at these frequencies when performing the other jump tests for the sake of comparability. While signature plots for the other jump test statistics might be instructive, they are not integral to the results of this paper and are omitted.

\subsection{Effects of Sampling Frequency on Jump Tests}

As discussed above, one can sample high-frequency stock price data at intervals of 5 to 11 minutes to diminish the effects of market microstructure noise. These sampled data contain most of the same information as the original data, only without market microstructure noise, so one could reasonably expect that a jump test performed on the same data sampled at two different frequencies would detect jumps on the same days. However, this is not the case, as the choice of sampling frequency affects both the number of jumps detected as well as the timing of those jumps for each of the tests described in Section 3.

To examine these effects, I performed each jump test on the stock price data described in Section 4 sampled at 1-minute, 5-minute, 10-minute, and 15-minute intervals. For the Ait-Sahalia Jacod test, instead of using the sample intervals above, I use a base sampling interval of 1 minute and $k$ values of 2,3 , and 4 . I make this distinction because they perform their test at sampling intervals of 5 seconds to 30 seconds in their paper, so 
sampling intervals longer than 1 minute seem inappropriate. The comparison of different values of $k$ for this test will provide similar insight to the comparison of different sampling frequencies for the other jump tests.

Table 1 presents the results of these tests performed at a .01 level of significance. The top four rows of each table contain the average percentage of jump days out of total days. It is worth noting that each test detects that more than one percent of the total days have jumps in them, using data sampled at all four intervals. Thus, the results presented here are not solely based on chance. The bottom six rows contain the average percentage of common jump days out of total days between each pair of sampling intervals. The results in Table 1 are averaged evenly across the ten stocks. Since the number of days of data available differs among the stocks in the sample, the results in Additional Table 2 in the Appendix are weighted by the number of days available for each stock. However, this distinction has a minimal effect on the results.

There is a distinct pattern in the amounts of jump days detected at different sampling frequencies for these jump tests. As the sampling interval increases from 1 to 15 minutes, there is a noticeable decrease in the number of jump days detected by each jump test. For the Ait-Sahalia Jacod test, the number of jump days detected decreases as $k$ increases and the base sampling interval is 1 minute.

The Barndorff-Nielsen Shephard tests detect an extremely high number of jumps with the 1-minute price data, suggesting that they perform particularly poorly in the presence of market microstructure noise. Although the Jiang Oomen and Lee Mykland tests do not exhibit as extreme a drop in the amount of jumps detected between the 1-minute and 5minute intervals, they still appear to be influenced by noise at the 1-minute level. The results of the Microstructure Noise Robust Jiang Oomen tests exhibit a similar pattern, indicating that there may be a problem with the noise correction at these sampling intervals.

While it is interesting that these tests find different numbers of jump days at different sampling frequencies, the most surprising result of this study is that the timing of these jump days varies between samples. The percentage of jump days out of total days that are common between two sampling frequencies is only a fraction of the percentage of jump days detected at each sampling frequency for every test and every pair of sampling frequencies. Furthermore, there does not appear to be any trend where high-frequency or low-frequency 
samples perform better among each other. This result is also surprising, as the tests are based on statistics that converge to their distributions as the number of observations approaches infinity, or as the sampling interval approaches zero.

\subsection{Incoherence Among Jump Tests}

Since these jump tests are all designed to measure the same phenomenon, extreme movements in asset prices, one might also reasonably expect that they would detect the same jumps. Tables 2 and 3 contain contingency matrices for the jump tests performed with prices sampled at 1-minute and 5-minute intervals, respectively. Each element of these matrices represents the average percentage of jump days out of total days that are common between the test on the row and the test on the column. The diagonal of each matrix contains the percentage of jump days out of total days detected by each test individually. The results are weighted evenly across stocks, rather than by the number of days of data available for each stock. Results weighted by the number of days of data available for each stock are presented in Additional Tables 3 and 4 in the Appendix, although this weighting has a minimal effect on the results.

There are several noteworthy results of this study. First, the Jiang Oomen Logarithmic and Ratio tests appear to detect the exact same jumps, as evidenced by Tables 2 and 3. The Microstructure Noise Robust Logarithmic and Ratio tests exhibit the same pattern. Furthermore, the Jiang Oomen Difference test is significantly more coherent with the Logarithmic and Ratio tests than it is with any other test. This pattern of coherence between tests by the same author appears in the results for the Microstructure Noise Robust tests and the Barndorff-Nielsen Shephard tests as well.

However, there appears to be little coherence among tests by different authors. Since the jump tests are based on statistics that converge to their distributions as the number of observations approaches infinity, one might expect the percentage of common jump days between these tests to increase as the sampling frequency increases. Surprisingly, that pattern does not appear in any significant sense between the 5-minute and 1-minute tables. The numbers are indeed higher in the 1-minute tables, particularly comparing the Jiang Oomen tests to the Barndorff-Nielsen Shephard and Lee Mykland tests, but this pattern is attributable to the higher percentage of jump days detected by these tests at this frequency. 


\section{Interpretation of Results}

While the authors of the jump tests described in Section 3 spent a great deal of time running simulations to test the accuracy and consistency of their tests, they all spent less time discussing the application of their tests to actual asset price data. Of course, the purpose of theses tests is to apply them to real stock data to learn more about their price paths. The results in Section 5 raise doubts about the validity and consistency of these tests when applied to stock price data.

This study demonstrates that the jump tests are susceptible to market microstructure noise, as evidenced by the Relative Jump volatility signature plot in Figure 3. This plot indicates that the contribution of jumps to total variance is $13 \%$ for the 1 -minute price data, whereas the contribution of jumps to total variance is only $7 \%$ for the 2 -minute data. Such a large drop in Relative Jump between price data sampled at 1-minute and 2-minute intervals implies that the Barndorff-Nielsen Shephard test statistics will be vastly different when performed on those two sets of data. This is a serious problem with the Barndorff-Nielsen Shephard tests that the authors might consider addressing in future revisions of the tests.

Furthermore, each jump test is inconsistent among price data sampled at different frequencies. This is an important finding of the study, as the problem cannot be easily resolved when applying the tests to real stock price data. Sampling prices at frequencies lower than the highest available appears to mitigate the effect of market microstructure noise, as evidenced by the results for the Barndorff-Nielsen Shephard tests. However, there is no clear pattern in Table 1 that suggests a solution to the inconsistencies in the jump tests due to the frequency at which price data are sampled.

These results cast doubt on the applicability of these jump tests to actual price data, and have major implications for authors who use the tests to detect jumps in the financial markets in their research. For instance, Tauchen and Zhou (2006) use the Barndorff-Nielsen $Z_{T P-\max }$ test to detect jumps in S\&P 500 Index prices sampled at 5-minute interval. They then derive the distribution of the jumps and use the time-varying jump volatility to forecast investment grade bond spread indices, finding that S\&P 500 jump volatility is a more effective predictor of bond spreads than interest rates, market volatility, and other risk factors. In a similar paper, Zhang, Zhou and Zhu (2005) use the Barndorff-Nielsen $Z_{T P-\max }$ 
test to detect jumps in individual equity prices sampled at a 5-minute interval and use the time-varying jump volatility to forecast Credit Default Swap spreads.

The results in Table 1 suggest that these authors might have found different results if they had chosen to sample price data at a different frequency. While this problem certainly does not preclude the usefulness of jump detection tests, it is worth taking into account when applying the tests to financial data. Moreover, these authors conduct their studies under the assumption of large and rare jumps. The results in Tables 2 and 3 suggest that the different jump tests are detecting different types of jump-like movements, be they large and rare jumps, small and frequent jumps, or simply noise. Thus, one should not assume that the model of large and rare jumps fully describes the jump-like phenomena in the financial markets.

One potential pitfall of my study is that the sampling frequencies chosen for the purpose of comparison in this paper may not all necessarily be appropriate for every jump test. For instance, Aït-Sahalia and Jacod (2008) perform their test on stock price data sampled at intervals of 5 seconds to 30 seconds, finding that the test statistic is more consistent with prices sampled at higher frequencies. This suggests that performing the tests on data sampled at lower frequencies will lead to inconsistencies in the results. Moreover, Lee and Mykland (2007) perform their test on price data sampled at a 15-minute interval, so the higher frequencies used in this paper may not be appropriate. Also, they perform their test at a .05 level of significance, so my choice of a .01 level of significance may be too far into the tail of the exponential distribution of the test statistic.

\section{Conclusion}

This paper applies recently developed tests for jumps in asset price movements to a set of highly liquid equities, with prices sampled at a variety of intervals. It finds that the choice of sampling interval impacts both the number of jumps as well as the timing of jumps detected by these tests. It also finds that among each other, these tests detect different amounts and timing of jumps when performed on the same data. These findings should motivate authors of jump detection tests to reconsider the applicability of their tests to stock price data and to address the impact of market microstructure noise and the choice of sampling frequency on 
their tests. Furthermore, authors who apply these tests to stock price data in their research should reconsider the validity of their results.

To further examine the impact of sampling frequency on jump detection tests, it will be necessary to obtain stock price data collected at sampling frequencies higher than 385 observations per day and perform the tests on these data, to determine whether the results of the tests converge as the number of observations per day approaches infinity. Beyond this, it will be necessary to conduct Monte Carlo simulations to see if this problem is a result of market factors or the properties of the tests. 


\section{Tables}

Throughout this section, the following abbreviations will be used:

$\mathrm{Z}_{\mathrm{TP}}$ : Barndorff-Nielsen Shephard test using Tri-Power Quarticity (3.1.8)

$\mathrm{Z}_{\mathrm{QP}}$ : Barndorff-Nielsen Shephard test using Quad-Power Quarticity (3.1.9)

$\mathrm{JO}_{\mathrm{D}}$ : Jiang Oomen Difference test (3.2.5)

$\mathrm{JO}_{\mathrm{L}}$ : Jiang Oomen Logarithmic test (3.2.6)

$\mathrm{JO}_{\mathrm{R}}$ : Jiang Oomen Ratio test (3.2.7)

$\mathrm{MNR}_{\mathrm{D}}$ : Microstructure Noise Robust Jiang Oomen Difference test (3.2.9)

$\mathrm{MNR}_{\mathrm{L}}$ : Microstructure Noise Robust Jiang Oomen Logarithmic test (3.2.10)

$\mathrm{MNR}_{\mathrm{R}}$ : Microstructure Noise Robust Jiang Oomen Ratio test (3.2.11)

LM: Lee Mykland test (3.3.3)

ASJ: Aït-Sahalia Jacod test (3.4.2) 


\section{Table 1*}

Inconsistency in the Number and Timing of Jumps Detected by Tests

Performed on Stock Prices Sampled at Different Intervals

\begin{tabular}{|c|cc|ccc|ccc|c|c|}
\hline & $\mathrm{Z}_{\mathrm{TP}}$ & $\mathrm{Z}_{\mathrm{QP}}$ & $\mathrm{JO}_{\mathrm{D}}$ & $\mathrm{JO}_{\mathrm{L}}$ & $\mathrm{JO}_{\mathrm{R}}$ & $\mathrm{MNR}_{\mathrm{D}}$ & $\mathrm{MNR}_{\mathrm{L}}$ & $\mathrm{MNR}_{\mathrm{R}}$ & $\mathrm{LM}$ & ASJ** \\
\hline $1 \mathrm{~m}$ jump days & .4929 & .5329 & .1785 & .1504 & .1504 & .1133 & .1614 & .1613 & .2015 & - \\
$5 \mathrm{~m}$ jump days & .0885 & .1018 & .1037 & .0852 & .0850 & .0909 & .0882 & .0881 & .0505 & .1530 \\
$10 \mathrm{~m}$ jump days & .0694 & .0761 & .0869 & .0624 & .0624 & .0829 & .0639 & .0638 & .0368 & .1228 \\
$15 \mathrm{~m}$ jump days & .0638 & .0698 & .0847 & .0509 & .0509 & .0837 & .0523 & .0521 & .0267 & .0982 \\
\hline $1 \mathrm{~m}, 5 \mathrm{~m}$ days & .0519 & .0652 & .0601 & .0490 & .0489 & .0372 & .0504 & .0504 & .0346 & - \\
$1 \mathrm{~m}, 10 \mathrm{~m}$ days & .0373 & .0449 & .0456 & .0342 & .0342 & .0289 & .0337 & .0336 & .0229 & - \\
$1 \mathrm{~m}, 15 \mathrm{~m}$ days & .0338 & .0406 & .0425 & .0271 & .0271 & .0279 & .0256 & .0255 & .0173 & - \\
$5 \mathrm{~m}, 10 \mathrm{~m}$ days & .0119 & .0151 & .0447 & .0369 & .0368 & .0393 & .0302 & .0302 & .0214 & .0786 \\
$5 \mathrm{~m}, 15 \mathrm{~m}$ days & .0085 & .0108 & .0370 & .0268 & .0267 & .0335 & .0214 & .0213 & .0150 & .0710 \\
$10 \mathrm{~m}, 15 \mathrm{~m}$ days & .0121 & .0142 & .0388 & .0267 & .0267 & .0369 & .0184 & .0183 & .0159 & .0676 \\
\hline
\end{tabular}

The rows titled $1 \mathrm{~m}$ jump days, $5 \mathrm{~m}$ jump days, $10 \mathrm{~m}$ jump days, and $15 \mathrm{~m}$ jump days contain the percentage of jump days out of total days for each sampling interval, averaged over the stocks in the sample. The rows titled $1 \mathrm{~m}, 5 \mathrm{~m}$ days; $1 \mathrm{~m}, 10 \mathrm{~m}$ days; $1 \mathrm{~m}, 15 \mathrm{~m}$ days; $5 \mathrm{~m}, 10 \mathrm{~m}$ days; $5 \mathrm{~m}, 15 \mathrm{~m}$ days; and $10 \mathrm{~m}, 15 \mathrm{~m}$ days contain the percentage of common jump days out of total days among each pair of sampling intervals, averaged over the stocks in the sample. All of the tests were performed at a .01 level of significance. The results presented in this table are averaged over the ten stocks in the sample, with no weighting for the number of days of data available for each stock. This means that the percentages are calculated individually for each stock, then they are summed and divided by ten. An alternative weighting scheme is presented in Additional Table 2 in the Appendix.

The jump tests all detect different numbers of jumps when performed on price data sampled at different frequencies, as evidenced by the top four rows of the table. Furthermore, the jump tests all detect different timings of jumps between different frequencies, as evidenced by the values in the bottom six rows, which are drastically lower than the values in the top four rows, meaning that there are few common days detected by each test among different sampling frequencies.

*: Explanations of the abbreviations used in the column headers can be found at the beginning of this section.

**: The Aït-Sahalia Jacod tests were performed at a 1-minute base sampling interval with $k=2,3$, 4. This choice is explained in Section 5.2. 
Table $2 *$

Incoherence among Tests by Different Authors in the Number and Timing

of Jumps Detected in Stock Price Data Sampled at a 1-Minute Interval

\begin{tabular}{|c|cc|ccc|ccc|c|c|}
\hline & $\mathrm{Z}_{\mathrm{TP}}$ & $\mathrm{Z}_{\mathrm{QP}}$ & $\mathrm{JO}_{\mathrm{D}}$ & $\mathrm{JO}_{\mathrm{L}}$ & $\mathrm{JO}_{\mathrm{R}}$ & $\mathrm{MNR}_{\mathrm{D}}$ & $\mathrm{MNR}_{\mathrm{L}}$ & $\mathrm{MNR}_{\mathrm{R}}$ & $\mathrm{LM}$ & ASJ** \\
\hline $\mathrm{Z}_{\mathrm{TP}}$ & $\mathbf{. 4 9 2 9}$ & & & & & & & & & \\
$\mathrm{Z}_{\mathrm{QP}}$ & .4917 & $\mathbf{. 5 3 2 9}$ & & & & & & & & \\
\hline $\mathrm{JO}_{\mathrm{D}}$ & .1051 & .1137 & $\mathbf{. 1 7 8 5}$ & & & & & & & \\
$\mathrm{JO}_{\mathrm{L}}$ & .0842 & .0916 & .1502 & $\mathbf{. 1 5 0 4}$ & & & & & & \\
$\mathrm{JO}_{\mathrm{R}}$ & .0842 & .0916 & .1502 & .1504 & $\mathbf{. 1 5 0 4}$ & & & & & \\
\hline $\mathrm{MNR}_{\mathrm{D}}$ & .0621 & .0674 & .1133 & .0987 & .0987 & $\mathbf{. 1 1 3 3}$ & & & & \\
$\mathrm{MNR}_{\mathrm{L}}$ & .0941 & .1018 & .1560 & .1433 & .1433 & .0948 & $\mathbf{. 1 6 1 4}$ & & & \\
$\mathrm{MNR}_{\mathrm{R}}$ & .0941 & .1017 & .1559 & .1433 & .1433 & .0948 & .1613 & $\mathbf{. 1 6 1 3}$ & & \\
\hline $\mathrm{LM}$ & .1148 & .1248 & .0651 & .0607 & .0607 & .0443 & .0609 & .0609 & $\mathbf{. 2 0 1 5}$ & \\
\hline $\mathrm{ASJ}^{* *}$ & .0634 & .0677 & .0229 & .0194 & .0194 & .0066 & .0241 & .0241 & .0117 & $\mathbf{. 0 9 3 1}$ \\
\hline
\end{tabular}

Each cell of this table contains the percentage of common jump days out of total days among the two jump tests on the row and column header, averaged over the stocks in the sample. The diagonal elements are the percentage of jump days out of total days detected by each test individually, averaged over the stocks in the sample. The jump tests are performed on price data sampled at a 1-minute interval. The results presented in this table are averaged over the ten stocks in the sample, with no weighting for the number of days of data available for each stock. This means that the percentages are calculated individually for each stock, then they are summed and divided by ten. An alternative weighting scheme is presented in Additional Table 3 in the Appendix.

Jump tests by the same author detect similar numbers of jump days, as well as similar timing of these jumps. However, jump tests by different authors detect different numbers of jump days with different timings, as evidenced by the non-diagonal values, which are drastically lower than the values on the diagonal.

*: Explanations of the abbreviations used in the row and column titles can be found at the beginning of this section.

**: The Ait-Sahalia Jacod tests were performed at a 1-minute base sampling interval with $k=2$. 
Table 3*

Incoherence among Tests by Different Authors in the Number and Timing

of Jumps Detected in Stock Price Data Sampled at a 5-Minute Interval

\begin{tabular}{|c|cc|ccc|ccc|c|c|}
\hline & $\mathrm{Z}_{\mathrm{TP}}$ & $\mathrm{Z}_{\mathrm{QP}}$ & $\mathrm{JO}_{\mathrm{D}}$ & $\mathrm{JO}_{\mathrm{L}}$ & $\mathrm{JO}_{\mathrm{R}}$ & $\mathrm{MNR}_{\mathrm{D}}$ & $\mathrm{MNR}_{\mathrm{L}}$ & $\mathrm{MNR}_{\mathrm{R}}$ & $\mathrm{LM}$ & $\mathrm{ASJ}^{* *}$ \\
\hline $\mathrm{Z}_{\mathrm{TP}}$ & $\mathbf{. 0 8 8 5}$ & & & & & & & & & \\
$\mathrm{Z}_{\mathrm{QP}}$ & .0868 & $\mathbf{. 1 0 1 8}$ & & & & & & & & \\
\hline $\mathrm{JO}_{\mathrm{D}}$ & .0273 & .0305 & $\mathbf{. 1 0 3 7}$ & & & & & & & \\
$\mathrm{JO}_{\mathrm{L}}$ & .0212 & .0239 & .0831 & $\mathbf{. 0 8 5 2}$ & & & & & & \\
$\mathrm{JO}_{\mathrm{R}}$ & .0212 & .0239 & .0830 & .0850 & $\mathbf{. 0 8 5 0}$ & & & & & \\
\hline $\mathrm{MNR}_{\mathrm{D}}$ & .0246 & .0274 & .0909 & .0733 & .0731 & $\mathbf{. 0 9 0 9}$ & & & & \\
$\mathrm{MNR}_{\mathrm{L}}$ & .0235 & .0265 & .0823 & .0741 & .0741 & .0698 & $\mathbf{. 0 8 8 2}$ & & & \\
$\mathrm{MNR}_{\mathrm{R}}$ & .0235 & .0265 & .0823 & .0741 & .0741 & .0698 & .0881 & $\mathbf{. 0 8 8 1}$ & & \\
\hline $\mathrm{LM}$ & .0152 & .0170 & .0185 & .0176 & .0176 & .0164 & .0170 & .0170 & $\mathbf{. 0 5 0 5}$ & \\
\hline $\mathrm{ASJ}^{* *}$ & .0014 & .0018 & .0054 & .0048 & .0048 & .0032 & .0058 & .0058 & .0012 & $\mathbf{. 0 1 8 2}$ \\
\hline
\end{tabular}

Each cell of this table contains the percentage of common jump days out of total days among the two jump tests on the row and column header, averaged over the stocks in the sample. The diagonal elements are the percentage of jump days out of total days detected by each test individually, averaged over the stocks in the sample. The jump tests are performed on price data sampled at a 5-minute interval. The results presented in this table are averaged over the ten stocks in the sample, with no weighting for the number of days of data available for each stock. This means that the percentages are calculated individually for each stock, then they are summed and divided by ten. An alternative weighting scheme is presented in Additional Table 4 in the Appendix.

Jump tests by the same author detect similar numbers of jump days, as well as similar timing of these jumps. However, jump tests by different authors detect different numbers of jump days with different timings, as evidenced by the non-diagonal values, which are drastically lower than the values on the diagonal.

*: Explanations of the abbreviations used in the row and column titles can be found at the beginning of this section.

**: The Aitt-Sahalia Jacod tests were performed at a 5-minute base sampling interval with $k=2$. 


\section{Figures}

Figure 1

Volatility Signature Plot Demonstrating the Impact of Market Microstructure Noise on Realized Variance when Calculated with High-Frequency Stock Price Data

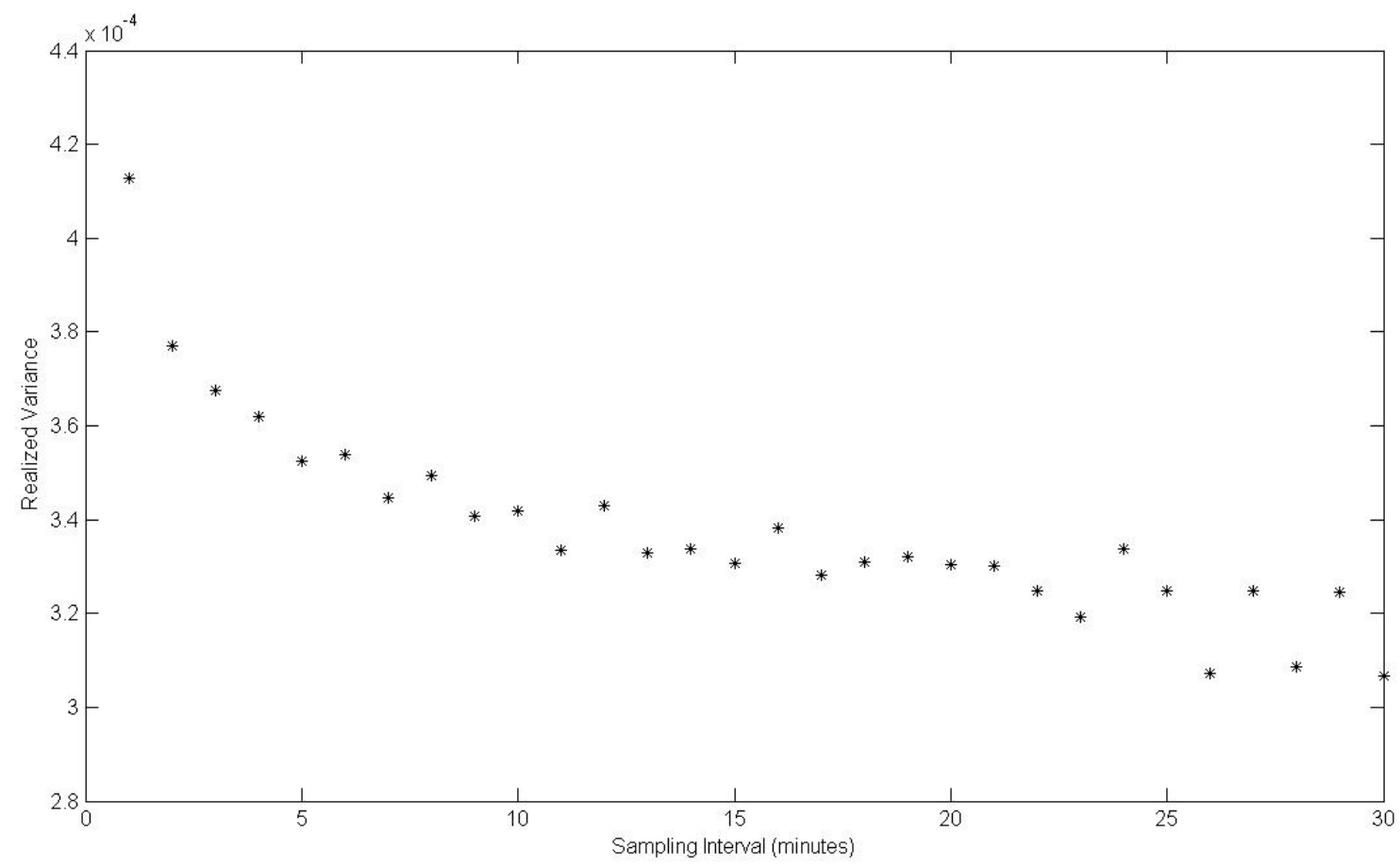

Each point on this graph represents the average value of Realized Variance (3.1.2) over the stocks in the sample, with prices sampled at the interval on the horizontal axis. The sampling intervals used for this plot range from 1 minute to 30 minutes. The theory behind this plot is explained in Section 5.1.

The downward sloping curve here is similar to the shape of the volatility signature plot for a highly liquid asset in Anderson et al. (1999), as expected. The sharp drop in the level of Realized Variance as the sampling interval increases from 1 to 5 minutes demonstrates the effect of market microstructure noise on the calculation. 
Figure 2

Volatility Signature Plot Demonstrating the Impact of Market Microstructure Noise on Bipower Variation when Calculated with High-Frequency Stock Price Data

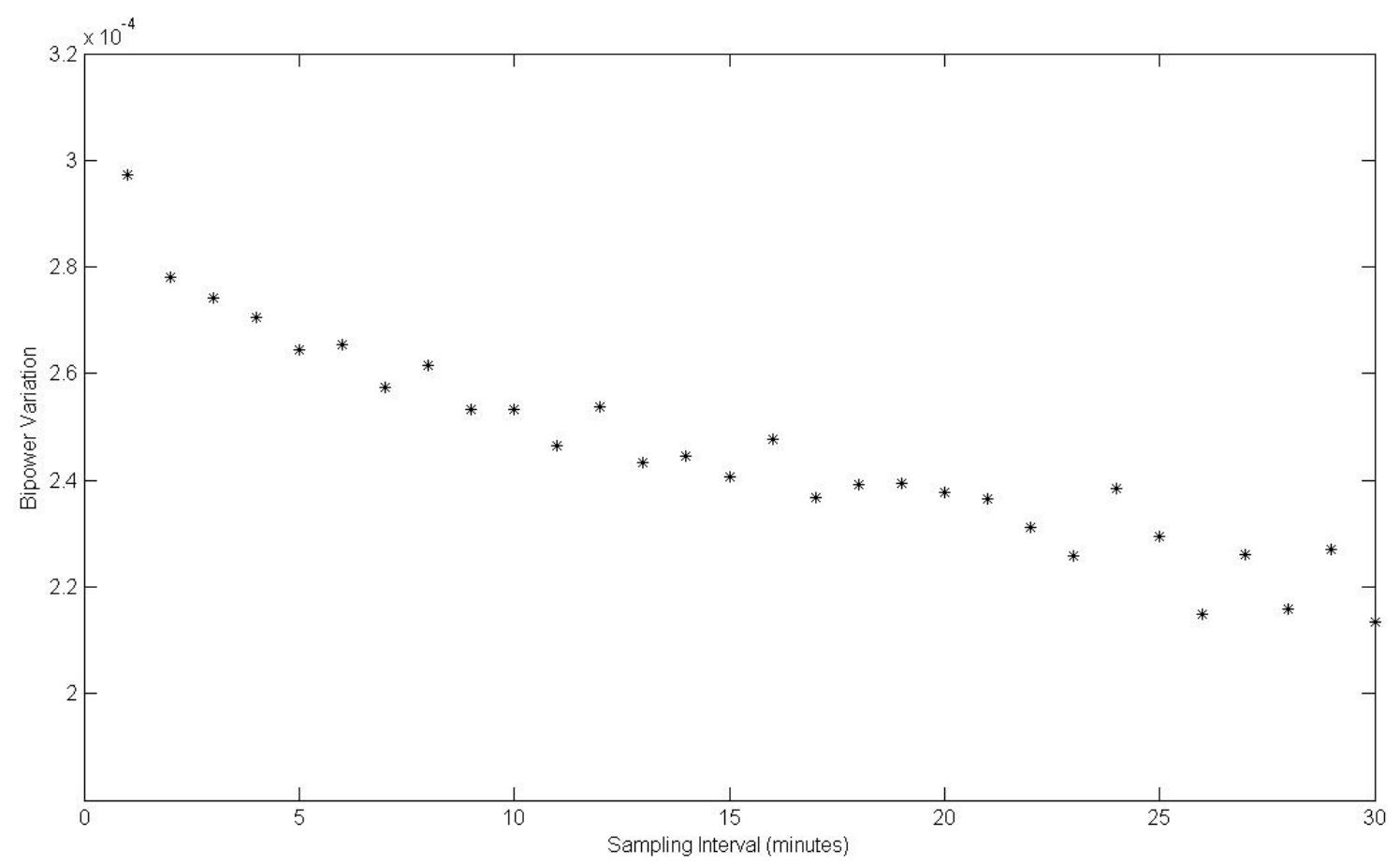

Each point on this graph represents the average value of Bipower Variation (3.1.3) over the stocks in the sample, with prices sampled at the interval on the horizontal axis. The sampling intervals used for this plot range from 1 minute to 30 minutes. The theory behind this plot is explained in Section 5.1.

The downward sloping curve here is similar to the shape of the volatility signature plot for a highly liquid asset in Anderson et al. (1999), as expected. The drop in the level of Bipower Variation as the sampling interval increases from 1 to 5 minutes demonstrates the effect of market microstructure noise on the calculation. 
Figure 3

Relative Jump Signature Plot Demonstrating the Impact of Market Microstructure Noise on Relative Jump when Calculated with High-Frequency Stock Price Data

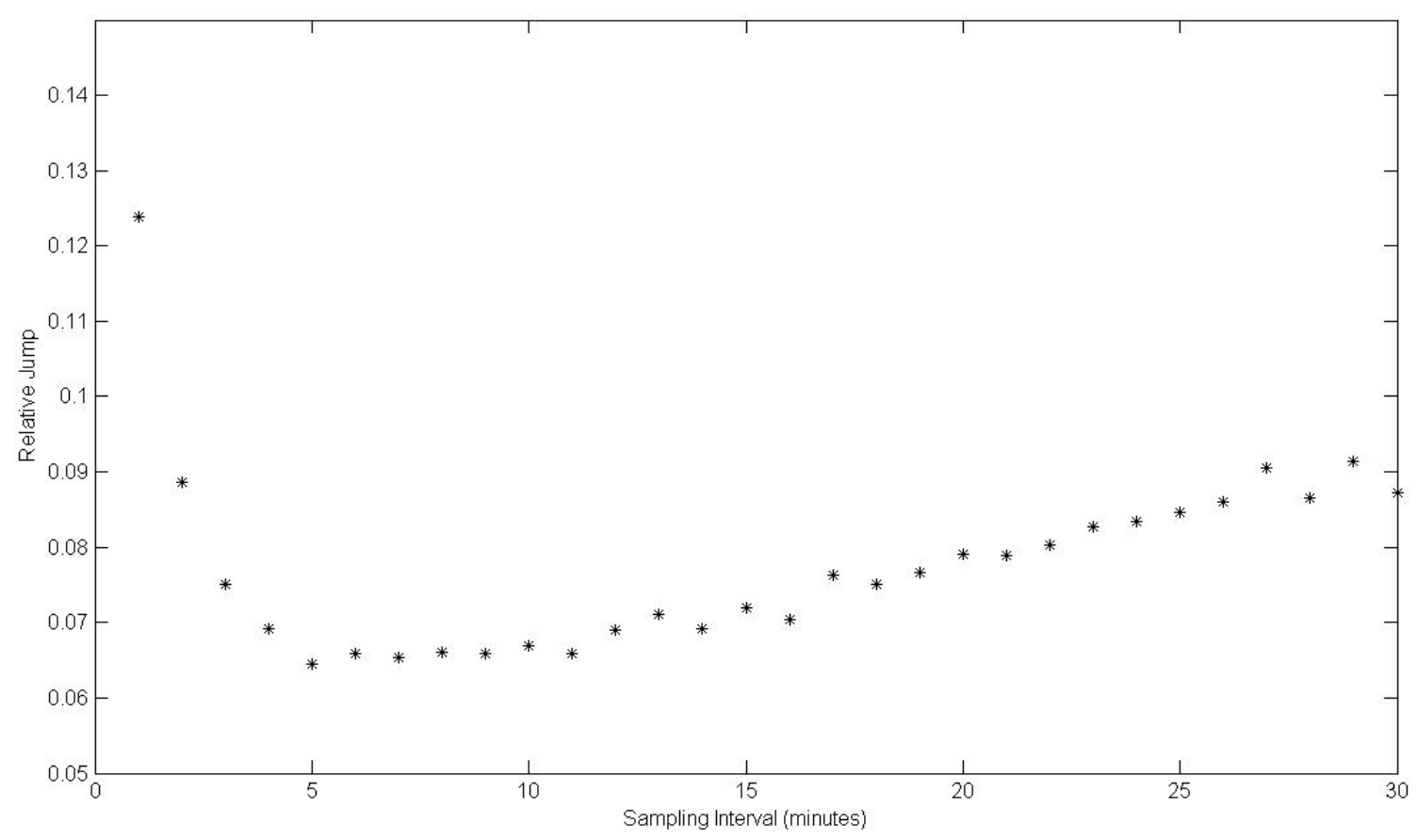

Each point on this graph represents the average value of Relative Jump (3.1.4) over the stocks in the sample, with prices sampled at the interval on the horizontal axis. The sampling intervals used for this plot range from 1 minute to 30 minutes. The theory behind this plot is explained in Section 5.1.

The sharp drop in the level of Relative Jump as the sampling interval increases from 1 to 5 minutes demonstrates the effect of market microstructure noise on the calculation. The relatively flat region in the plot between the 5-minute and 11-minute sampling intervals suggests that is appropriate to sample prices at these intervals to mitigate the effects of market microstructure noise when performing the Barndorff-Nielsen Shephard jump tests. 


\section{References}

Aït-Sahalia, Y. and J. Jacod. (2008). Testing for Jumps in a Discretely Observed Process. Annals of Statistics, forthcoming.

Andersen, T., T. Bollerslev, and F. Diebold. (2004). Some Like It Smooth, and Some Like It Rough: Untangling Continuous and Jump Components in Measuring, Modeling and Forecasting Asset Return Volatility. Working paper, Duke University.

Andersen, T., T. Bollerslev, F. Diebold, and P. Labys. (1999). (Understanding, Optimizing, Using and Forecasting) Realized Volatility and Correlation. Manuscript.

Barndorff-Nielsen, O. and N. Shephard. (2005). Variation, jumps, market frictions, and high frequency data in financial econometrics. Advances in Economics and Econometrics: Theory and Applications, Ninth World Congress (Econometric Society Monographs). Cambridge University Press, 2007. 328-372.

Barndorff-Nielsen, O. and N. Shephard. (2006). Econometrics of Testing for Jumps in Financial Economics Using Bipower Variation. Journal of Financial Econometrics, $4(1), 1-30$.

Cont, R. and P. Tankov. (2004). Financial Modelling with Jump Processes. Boca Raton, Florida: CRC Press, 2004.

Fama, E. (1965). The Behavior of Stock-Market Prices. Journal of Business, 38 (1), 34105.

Fama, E. and K. French. (1988). Permanent and Temporary Components of Stock Prices. Journal of Political Economy, 96 (2), 246-273.

Huang, X. and G. Tauchen. (2005). The Relative Contribution of Jumps to Total Price Variance. Journal of Financial Econometrics, 3 (4), 456-499.

Jiang, G. and R. Oomen. (2007). Testing for Jumps When Asset Prices Are Observed with Noise: a "Swap Variance" Approach. Journal of Econometrics, forthcoming.

Lee, S. and P. Mykland. (2008). Jumps in Financial Markets: A New Nonparametric Test and Jump Dynamics. Review of Financial Studies, 21(6), 2535-2563.

Levy, H. and T. Post. Investments. Essex, England: Pearson Education Limited, 2005. 498501.

Merton, R.C. (1971). Optimum consumption and portfolio rules in a continuous-time model. Journal of Economic Theory, 3, 373-413. 
Merton, R.C. (1976). Option pricing when underlying stock returns are discontinuous. Journal of Financial Economics, 3, 125-144.

Roll, R. (1984). A Simple Implicit Measure of the Effective Bid-Ask Spread in an Efficient Market. Journal of Finance, 4, 1127-1139.

Tauchen, G. and H. Zhou. (2006). Realized Jumps on Financial Markets and Predicting Credit Spreads. Working paper, Duke University.

Zhang, B.Y., H. Zhou, and H. Zhu. (2008). Explaining Credit Default Swap Spreads with the Equity Volatility and Jump Risks of Individual Firms. Review of Financial Studies, forthcoming. 


\section{Appendix}

Throughout this section, the following abbreviations will be used:

$\mathrm{Z}_{\mathrm{TP}}$ : Barndorff-Nielsen Shephard test using Tri-Power Quarticity (3.1.8)

$\mathrm{Z}_{\mathrm{QP}}$ : Barndorff-Nielsen Shephard test using Quad-Power Quarticity (3.1.9)

$\mathrm{JO}_{\mathrm{D}}$ : Jiang Oomen Difference test (3.2.5)

$\mathrm{JO}_{\mathrm{L}}$ : Jiang Oomen Logarithmic test (3.2.6)

$\mathrm{JO}_{\mathrm{R}}$ : Jiang Oomen Ratio test (3.2.7)

$M_{N R_{D}}$ : Microstructure Noise Robust Jiang Oomen Difference test (3.2.9)

$\mathrm{MNR}_{\mathrm{L}}$ : Microstructure Noise Robust Jiang Oomen Logarithmic test (3.2.10)

$\mathrm{MNR}_{\mathrm{R}}$ : Microstructure Noise Robust Jiang Oomen Ratio test (3.2.11)

LM: Lee Mykland test (3.3.3)

ASJ: Aït-Sahalia Jacod test (3.4.2) 
Additional Table 1

Sample of Equities

\begin{tabular}{|c|c|c|c|c|}
\hline Company & Ticker Symbol & Exchange & Dates & Days in Sample \\
\hline ExxonMobil & XOM & NYSE & $12 / 1 / 99-1 / 24 / 08$ & 2026 \\
General Electric & GE & NYSE & $4 / 9 / 97-12 / 31 / 07$ & 2670 \\
Microsoft & MSFT & NASDAQ & $4 / 16 / 97-1 / 24 / 08$ & 2683 \\
AT\&T & T & NYSE & $4 / 9 / 97-1 / 24 / 98$ & 2680 \\
Procter \& Gamble & PG & NYSE & $4 / 9 / 97-1 / 24 / 08$ & 2686 \\
Chevron & CVX & NYSE & $10 / 10 / 01-1 / 24 / 08$ & 1566 \\
Johnson \& Johnson & JNJ & NYSE & $4 / 9 / 97-1 / 24 / 08$ & 2685 \\
Bank of America & BAC & NYSE & $4 / 9 / 97-1 / 24 / 08$ & 2685 \\
Cisco Systems & CSCO & NASDAQ & $4 / 16 / 97-1 / 24 / 08$ & 2683 \\
Altria Group & MO & NYSE & $4 / 9 / 97-1 / 24 / 08$ & 2685 \\
\hline
\end{tabular}




\section{Additional Table 2*}

Inconsistency in the Number and Timing of Jumps Detected by Tests Performed on

Stock Prices Sampled at Different Intervals - Alternative Weighting of Results

\begin{tabular}{|c|cc|ccc|ccc|c|c|}
\hline & $\mathrm{Z}_{\mathrm{TP}}$ & $\mathrm{Z}_{\mathrm{QP}}$ & $\mathrm{JO}_{\mathrm{D}}$ & $\mathrm{JO}_{\mathrm{L}}$ & $\mathrm{JO}_{\mathrm{R}}$ & $\mathrm{MNR}_{\mathrm{D}}$ & $\mathrm{MNR}_{\mathrm{L}}$ & $\mathrm{MNR}_{\mathrm{R}}$ & $\mathrm{LM}$ & $\mathrm{ASJ}^{* *}$ \\
\hline $1 \mathrm{~m}$ jump days & .5048 & .5457 & .1787 & .1502 & .1502 & .1121 & .1616 & .1615 & .2026 & - \\
$5 \mathrm{~m}$ jump days & .0903 & .1041 & .1054 & .0862 & .0860 & .0921 & .0894 & .0893 & .0516 & .1575 \\
$10 \mathrm{~m}$ jump days & .0704 & .0774 & .0881 & .0629 & .0628 & .0839 & .0647 & .0646 & .0373 & .1266 \\
$15 \mathrm{~m}$ jump days & .0647 & .0708 & .0856 & .0517 & .0517 & .0846 & .0526 & .0524 & .0274 & .1016 \\
\hline $1 \mathrm{~m}, 5 \mathrm{~m}$ days & .0539 & .0679 & .0608 & .0493 & .0491 & .0370 & .0510 & .0510 & .0354 & - \\
$1 \mathrm{~m}, 10 \mathrm{~m}$ days & .0385 & .0464 & .0462 & .0344 & .0344 & .0289 & .0342 & .0341 & .0235 & - \\
$1 \mathrm{~m}, 15 \mathrm{~m}$ days & .0347 & .0417 & .0427 & .0273 & .0273 & .0275 & .0255 & .0254 & .0179 & - \\
$5 \mathrm{~m}, 10 \mathrm{~m}$ days & .0123 & .0157 & .0454 & .0373 & .0372 & .0399 & .0307 & .0307 & .0220 & .0822 \\
$5 \mathrm{~m}, 15 \mathrm{~m}$ days & .0087 & .0111 & .0376 & .0272 & .0271 & .0340 & .0216 & .0215 & .0154 & .0740 \\
$10 \mathrm{~m}, 15 \mathrm{~m}$ days & .0125 & .0145 & .0392 & .0271 & .0271 & .0372 & .0187 & .0186 & .0163 & .0703 \\
\hline
\end{tabular}

The rows titled $1 \mathrm{~m}$ jump days, $5 \mathrm{~m}$ jump days, $10 \mathrm{~m}$ jump days, and $15 \mathrm{~m}$ jump days contain the percentage of jump days out of total days for each sampling interval, averaged over the stocks in the sample. The rows titled $1 \mathrm{~m}, 5 \mathrm{~m}$ days; $1 \mathrm{~m}, 10 \mathrm{~m}$ days; $1 \mathrm{~m}, 15 \mathrm{~m}$ days; $5 \mathrm{~m}, 10 \mathrm{~m}$ days; $5 \mathrm{~m}, 15 \mathrm{~m}$ days; and $10 \mathrm{~m}, 15 \mathrm{~m}$ days contain the percentage of common jump days out of total days among each pair of sampling intervals, averaged over the stocks in the sample. All of the tests were performed at a .01 level of significance. The results presented in this table are averaged over the ten stocks in the sample, weighted for the number of days of data available for each stock. This means that the numbers of jump days are calculated individually for each stock, then they are summed and divided by the sum of the trading days available for each stock $(25,049)$.

The jump tests all detect different numbers of jumps when performed on price data sampled at different frequencies, as evidenced by the top four rows of the table. Furthermore, the jump tests all detect different timings of jumps between different frequencies, as evidenced by the values in the bottom six rows, which are drastically lower than the values in the top four rows, meaning that there are few common days detected by each test among different sampling frequencies.

*: Explanations of the abbreviations used in the column headers can be found at the beginning of this section.

**: The Aït-Sahalia Jacod tests were performed at a 1-minute base sampling interval with $k=2,3$, 4. This choice is explained in Section 5.2. 


\section{Additional Table $3 *$}

Incoherence among Tests by Different Authors in the Number and Timing of Jumps Detected in Stock Price Data Sampled at a 1-Minute Interval - Alternative Weighting of Results

\begin{tabular}{|c|cc|ccc|ccc|c|c|}
\hline & $\mathrm{Z}_{\mathrm{TP}}$ & $\mathrm{Z}_{\mathrm{QP}}$ & $\mathrm{JO}_{\mathrm{D}}$ & $\mathrm{JO}_{\mathrm{L}}$ & $\mathrm{JO}_{\mathrm{R}}$ & $\mathrm{MNR}_{\mathrm{D}}$ & $\mathrm{MNR}_{\mathrm{L}}$ & $\mathrm{MNR}_{\mathrm{R}}$ & $\mathrm{LM}$ & $\mathrm{ASJ}^{* *}$ \\
\hline $\mathrm{Z}_{\mathrm{TP}}$ & $\mathbf{. 5 0 4 8}$ & & & & & & & & & \\
$\mathrm{Z}_{\mathrm{QP}}$ & .5037 & $\mathbf{. 5 4 5 7}$ & & & & & & & & \\
\hline $\mathrm{JO}_{\mathrm{D}}$ & .1066 & .1153 & $\mathbf{. 1 7 8 7}$ & & & & & & & \\
$\mathrm{JO}_{\mathrm{L}}$ & .0850 & .0926 & .1500 & $\mathbf{. 1 5 0 2}$ & & & & & & \\
$\mathrm{JO}_{\mathrm{R}}$ & .0850 & .0926 & .1500 & .1502 & $\mathbf{. 1 5 0 2}$ & & & & & \\
\hline $\mathrm{MNR}_{\mathrm{D}}$ & .0620 & .0672 & .1121 & .0974 & .0974 & $\mathbf{. 1 1 2 1}$ & & & & \\
$\mathrm{MNR}_{\mathrm{L}}$ & .0955 & .1034 & .1562 & .1431 & .1431 & .0936 & $\mathbf{. 1 6 1 6}$ & & & \\
$\mathrm{MNR}_{\mathrm{R}}$ & .0954 & .1033 & .1561 & .1430 & .1430 & .0936 & .1615 & $\mathbf{. 1 6 1 5}$ & & \\
\hline $\mathrm{LM}$ & .1162 & .1263 & .0658 & .0612 & .0612 & .0442 & .0614 & .0614 & $\mathbf{. 2 0 2 6}$ & \\
\hline $\mathrm{ASJ}^{* *}$ & .0670 & .0714 & .0232 & .0196 & .0196 & .0067 & .0244 & .0244 & .0119 & $\mathbf{. 0 9 6 2}$ \\
\hline
\end{tabular}

Each cell of this table contains the percentage of common jump days out of total days among the two jump tests on the row and column header, averaged over the stocks in the sample. The diagonal elements are the percentage of jump days out of total days detected by each test individually, averaged over the stocks in the sample. The jump tests are performed on price data sampled at a 1-minute interval. The results presented in this table are averaged over the ten stocks in the sample, with no weighting for the number of days of data available for each stock. This means that the percentages are calculated individually for each stock, then they are summed and divided by ten. An alternative weighting scheme is presented in Additional Table 3 in the Appendix.

Jump tests by the same author detect similar numbers of jump days, as well as similar timing of these jumps. However, jump tests by different authors detect different numbers of jump days with different timings, as evidenced by the non-diagonal values, which are drastically lower than the values on the diagonal.

*: Explanations of the abbreviations used in the row and column titles can be found at the beginning of this section.

**: The Ait-Sahalia Jacod tests were performed at a 1-minute base sampling interval with $k=2$. 


\section{Additional Table 4*}

Incoherence among Tests by Different Authors in the Number and Timing of Jumps Detected in Stock Price Data Sampled at a 5-Minute Interval - Alternative Weighting of Results

\begin{tabular}{|c|cc|ccc|ccc|c|c|}
\hline & $\mathrm{Z}_{\mathrm{TP}}$ & $\mathrm{Z}_{\mathrm{QP}}$ & $\mathrm{JO}_{\mathrm{D}}$ & $\mathrm{JO}_{\mathrm{L}}$ & $\mathrm{JO}_{\mathrm{R}}$ & $\mathrm{MNR}_{\mathrm{D}}$ & $\mathrm{MNR}_{\mathrm{L}}$ & $\mathrm{MNR}_{\mathrm{R}}$ & $\mathrm{LM}$ & $\mathrm{ASJ}^{* *}$ \\
\hline $\mathrm{Z}_{\mathrm{TP}}$ & $\mathbf{. 0 9 0 3}$ & & & & & & & & & \\
$\mathrm{Z}_{\mathrm{QP}}$ & .0885 & $\mathbf{. 1 0 4 1}$ & & & & & & & & \\
\hline $\mathrm{JO}_{\mathrm{D}}$ & .0280 & .0314 & $\mathbf{. 1 0 5 4}$ & & & & & & & \\
$\mathrm{JO}_{\mathrm{L}}$ & .0216 & .0244 & .0842 & $\mathbf{. 0 8 6 2}$ & & & & & & \\
$\mathrm{JO}_{\mathrm{R}}$ & .0216 & .0244 & .0840 & .0860 & $\mathbf{. 0 8 6 0}$ & & & & & \\
\hline $\mathrm{MNR}_{\mathrm{D}}$ & .0252 & .0281 & .0921 & .0739 & .0738 & $\mathbf{. 0 9 2 1}$ & & & & \\
$\mathrm{MNR}_{\mathrm{L}}$ & .0240 & .0271 & .0834 & .0751 & .0750 & .0704 & $\mathbf{. 0 8 9 4}$ & & & \\
$\mathrm{MNR}_{\mathrm{R}}$ & .0240 & .0271 & .0834 & .0751 & .0750 & .0704 & .0893 & $\mathbf{. 0 8 9 3}$ & & \\
\hline $\mathrm{LM}$ & .0154 & .0173 & .0191 & .0182 & .0182 & .0170 & .0176 & .0176 & $\mathbf{. 0 5 1 6}$ & \\
\hline $\mathrm{ASJ}^{* *}$ & .0014 & .0018 & .0055 & .0050 & .0050 & .0032 & .0059 & .0059 & .0013 & $\mathbf{. 0 1 8 4}$ \\
\hline
\end{tabular}

Each cell of this table contains the percentage of common jump days out of total days among the two jump tests on the row and column header, averaged over the stocks in the sample. The diagonal elements are the percentage of jump days out of total days detected by each test individually, averaged over the stocks in the sample. The jump tests are performed on price data sampled at a 5-minute interval. The results presented in this table are averaged over the ten stocks in the sample, with no weighting for the number of days of data available for each stock. This means that the percentages are calculated individually for each stock, then they are summed and divided by ten. An alternative weighting scheme is presented in Additional Table 4 in the Appendix.

Jump tests by the same author detect similar numbers of jump days, as well as similar timing of these jumps. However, jump tests by different authors detect different numbers of jump days with different timings, as evidenced by the non-diagonal values, which are drastically lower than the values on the diagonal.

*: Explanations of the abbreviations used in the row and column titles can be found at the beginning of this section.

**: The Aït-Sahalia Jacod tests were performed at a 5-minute base sampling interval with $k=2$. 